\title{
THE HOMOTOPY CLASSIFICATION OF SELF-MAPS OF INFINITE QUATERNIONIC PROJECTIVE SPACE
}

\author{
By GUIDO MISLIN
}

[Received 6 May 1986]

We say that a self-map $f: M P^{\infty} \rightarrow \boxplus P^{\infty}$ of infinite quaternionic projective space has degree $k, \operatorname{deg}(f)=k$, if the induced map of $\Omega H P^{\infty} \simeq S^{3}$ is of degree $k$ in the usual sense. It is well known that $\operatorname{deg}(f)$ is zero or an odd square integer [6]. The self-maps of $H P^{\infty}=B S^{3}$ which are induced from Lie groups endomorphisms of $S^{3}$ are easily seen to be of degree zero or one. Using localization techniques and methods from étale homotopy theory, D. Sullivan was able to construct self-maps of $M P^{\infty}$ of any given odd square degree [14]. To complete the picture, we present a proof of the following theorem.

Classification Theorem. Self-maps of $H P^{\infty}$ are classified up to homotopy by their degree.

Our proof relies on recent work of $H$. Miller concerning the generalized Sullivan conjecture [13] and a beautiful application thereof by W. Dwyer [5]. We need some notation to describe Dwyer's result. Let $\rho: H \rightarrow G$ be a Lie group homomorphism and put

$$
C(\rho)=\left\{g \in G \mid g \rho(x) g^{-1}=\rho(x) \text { for all } x \in H\right\} \subset G,
$$

the centralizer of $\rho$ in $G$. Define map $(B H, B G)$ to be the space of all free maps $B H \rightarrow B G$, and let map $(B H, B G)_{\rho}$ denote the component of map $(B H, B G)$ containing $B \rho$. The homomorphism $C(\rho) \times H \rightarrow G$, given by $(g, x) \rightarrow g \rho(x)$, gives rise to a map

$$
\Phi(\rho): B C(\rho) \rightarrow \operatorname{map}(B H, B G)_{\rho}
$$

which has the following property.

Proposition (W. Dwyer). Let $P$ be a finite p-group and $G$ a compact connected Lie group. Let $R \subset \operatorname{Hom}(P, G)$ denote a set of representatives for the conjugacy classes of homomorphisms $P \rightarrow G$. Then the following holds.

(i) $\operatorname{map}(B P, B G)=\coprod_{\rho \in R} \operatorname{map}(B P, B G)_{\rho}$

(ii) $\Phi(\rho): B C(\rho) \rightarrow \operatorname{map}(B P, B G)_{\rho}$ induces an isomorphism of fundamental groups and of homology groups with $\mathbf{Z} / p \mathbf{Z}$-coefficients.

In Section 1 we analyze restrictions of maps $B S^{3} \rightarrow B S^{3}$ to subspaces of 
the form $B \pi \subset B S^{3}, \pi \subset S^{3}$ a finite $p$-subgroup. This discussion leads to a reduction of the proof of the Classification Theorem to statements involving only odd primes and one for the prime two; the resulting problem (1.7) is dealt with in Section 3. Its solution involves a reconstruction of the classifying space of a finite group from the classifying spaces of its 2-subgroups. This construction gives rise to a spectral sequence relating various mapping spaces. The convergence problem for this spectral sequence is handled by a computation of derived functors of inverse limit functors, completing the proof of the Classification Theorem. In an Appendix we present a new proof of the fact that every self map of $B S^{3}$ has degree zero or an odd square integer.

It is a pleasure to thank Stefan Jackowski for valuable discussions concerning Section 2 .

\section{Restrictions to finite $p$-subgronps}

We will henceforth write $B S^{3}$ for $\mathbb{A} P^{\infty}$ and use the notation $f \mid B \pi$ for the restriction of a map $f: B S^{3} \rightarrow X$ to $B \pi \subset B S^{3}, \pi \subset S^{3}$ a finite subgroup.

LEMMA 1.1. Let $f, g: B S^{3} \rightarrow B S^{3}$ be two maps of the same degree. Then $f|B \pi \simeq g| B \pi$ for every finite $p$-subgroup $\pi$ of $S^{3}$ and every prime $p$.

Proof. By Proposition (i) we know that $f \mid B \pi \approx B \rho(f)$ and $g \mid B \pi \approx$ $B \rho(g)$, where $\rho(f), \rho(g): \pi \rightarrow S^{3}$ are certain representations of $\pi$, uniquely determined up to conjugation. Recall that for representable complex $K$-theory $K^{*}$ and $G$ a compact Lie group, $K^{*} B G \cong R(G)^{*}$, the $I(G)$-adic completion of the complex representation ring $R(G)$ of $G$. If $G$ is a finite $p$-group or a compact and connected Lie group, then the completion map $R(G) \rightarrow R(G)^{-}$is known to be injective. By a result of $S$. Feder and S. Gitler (cf. [6]) we know that two self-maps of $B S^{3}$ of the same degree induce the same map in $K$-theory. Thus, if we write $\phi$ for $K^{*}(f)=K^{*}(g)$, we obtain a commutative diagram

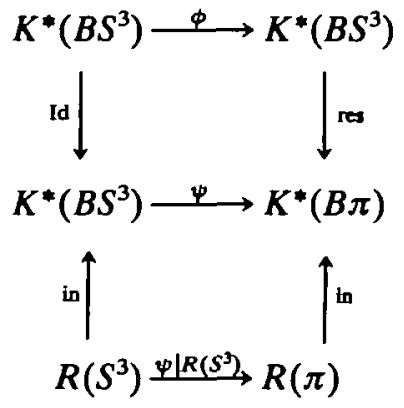


where $K^{*}(B \rho(f))=\psi=K^{*}(B \rho(g))$ and thus $\rho(f)^{*}=\psi \mid R\left(S^{3}\right)=\rho(g)^{*}$. If we denote by $I \in R\left(S^{3}\right)$ the class of the identity representation $S^{3} \rightarrow S U(2) \subset U(2)$, then $\rho(f)^{*} I=[\rho(f)]$, the conjugacy class of the composite map $\rho(f): \pi \rightarrow S U(2) \subset U(2)$. Therefore, since $\rho(f)^{*}=$ $\rho(g)^{*}$, it follows that $\rho(f)$ and $\rho(g)$ are conjugate in $U(2)$ and hence also in $S U(2)$. Consequently, $B \rho(f) \simeq B \rho(g)$ or $f|B \pi \simeq g| B \pi$.

Lemma 1.1 enables us to give a short proof of the following special case of the Classification Theorem, which had earlier been proved by A. Zabrodsky using different methods [16].

LeMMA 1.2. If $f: B S^{3} \rightarrow B S^{3}$ has degree zero, then $f$ is homotopic to a constant map.

Namely, observe that (1.1) implies that if $\operatorname{deg}(f)=0$, then $f \mid B \pi \simeq$ const. for every finite $p$-subgroup $\pi \subset S^{3}$. Hence $f$ is homotopic to a constant map by $[10,3.3]$.

To prove the Classification Theorem it suffices in view of (1.1) to show that the restriction map

$$
\Lambda:\left[B S^{3}, B S^{3}\right] \rightarrow \prod\left[B \pi, B S^{3}\right],
$$

is injective, where the product is taken over all finite $p$-subgroups $\pi$ of $S^{3}$ for all primes $p$. It is convenient to separate this problem into a problem at odd primes and one for the prime two. For this purpose consider Sullivan's profinite completion $\left(B S^{3}\right)^{\wedge}$ of $B S^{3}$. The homotopy fibration sequence $B S^{3} \rightarrow\left(B S^{3}\right)^{\wedge} \rightarrow K(\hat{Z} / Z, 4)$ shows that

$$
\left[B S^{3}, B S^{3}\right] \rightarrow\left[B S^{3},\left(B S^{3}\right)^{\hat{1}}\right]
$$

is injective, because $\left[B S^{3}, K(Z \mathbf{Z}, 3)\right]=0$. If we decompose $\left(B S^{3}\right)^{\wedge}$ into a product of $p$-profinite completions $\left(B S^{3}\right)_{p}$ then clearly, for every $p$-group $\pi$

$$
\left[B \pi, B S^{3}\right] \cong\left[B \pi,\left(B S^{3}\right)^{\hat{1}}\right] \cong\left[B \pi,\left(B S^{3}\right)_{p}^{\hat{p}}\right] .
$$

We define for a map $\phi: B S^{3} \rightarrow\left(B S^{3}\right)_{p}^{\hat{p}}$ the degree, $\operatorname{deg}(\phi) \in \mathbb{Z}_{p}$, to be the degree of the induced self-map of $\Omega\left(B S^{3}\right)_{p} \simeq\left(S^{3}\right)_{p}$.

Because of (1.2) and as already remarked in the introduction (cf. [6]), a self-map of $B S^{3}$ which is not homotopic to a constant map, has necessarily an odd degree. In view of (1.3), the Classification Theorem is thus a consequence of the following theorem.

THEOREM 1.4.

(i) Let $p$ be an odd prime. The map induced by restriction to all finite $p$-subgroups $\pi$ of $S^{3}$

$$
\left[B S^{3},\left(B S^{3}\right)_{p}^{\hat{p}}\right] \rightarrow \prod\left[B \pi,\left(B S^{3}\right)_{p}^{\hat{p}}\right]
$$

is injective. 
(ii) Let $U\left[B S^{3},\left(B S^{3}\right)_{2}\right] \subset\left[B S^{3},\left(B S^{3}\right)_{2}\right]$ denote the subset consisting of maps with degree units in $\hat{\mathbf{Z}}_{2}$. The map induced by restriction to all finite 2-subgroups $\pi$ of $S^{3}$

$$
U\left[B S^{3},\left(B S^{3}\right)_{2}\right] \rightarrow \prod\left[B \pi,\left(B S^{3}\right)_{2}^{\hat{2}}\right]
$$

is injective.

Proof of (i). If $p$ denotes an odd prime, $p$ does not divide the order of the Weyl group of $S^{3}$. A result of Wojtkowiak's [15, Corollary 3] asserts that in this case the restriction map

$$
\left[B S^{3},\left(B S^{3}\right)_{p}^{\hat{p}}\right] \rightarrow \prod_{n}\left[B\left(\mathbb{Z} / p^{n} \mathbb{Z}\right),\left(B S^{3}\right)_{p}^{\hat{p}}\right]
$$

is injective, where the subgroups $\mathbb{Z} / p^{n} \mathbb{Z} \subset S^{3}$ run through the family of finite $p$-subgroups of a fixed maximal torus $S^{1}$ of $S^{3}$.

Part (ii) of (1.4) will follow from Theorem 1.7 below; a different proof of (i) could easily be established along the lines of the proof of (ii).

LEMMA 1.5. Let $\phi: B S^{3} \rightarrow\left(B S^{3}\right)_{2}$ be a map of degree a unit in $\hat{\mathbb{Z}}_{2}$. If $\pi \subset S^{3}$ is a finite 2-subgroup, then $\phi \mid B \pi \approx(B \rho)_{2}$, where $\rho: \pi \rightarrow S^{3}$ is a faithful representation uniquely determined up to conjugation by $\phi$.

Proof. Since $\pi$ is a 2-group we infer that $\left[B \pi, B S^{3}\right] \rightarrow\left[B \pi,\left(B S^{3}\right)_{2}\right]$ is bijective and therefore, by Proposition (i), $\phi \mid B \pi \simeq(B \rho)_{2}$ where $\rho: \pi \rightarrow S^{3}$ is uniquely determined by $\phi$ up to conjugation. Since $\pi$ is a finite subgroup of $S^{3}$, its Tate cohomology is periodic of period four and $H^{4}(B \pi ; \mathbb{Z}) \cong \mathbb{Z} /|\pi| \mathbb{Z}$. Moreover, the restriction map $H^{4}\left(B S^{3} ; \mathbb{Z}\right) \rightarrow$ $H^{4}(B \pi ; \mathbb{Z})=H^{4}\left(B \pi ; \mathbb{Z}_{2}\right)$ is surjective. The assumption on the degree of $\phi$ implies therefore that

$$
(\phi \mid B \pi)^{*}: H^{4}\left(B S^{3} ; \hat{\mathbb{Z}}_{2}\right) \rightarrow H^{4}\left(B \pi ; \hat{\mathbf{Z}}_{2}\right)
$$

is surjective. Consequently, $(B \rho)^{*}: H^{4}\left(B S^{3} ; \mathbb{Z}\right) \rightarrow H^{4}(B \pi ; \mathbb{Z})=\mathbb{Z} /|\pi| \mathbb{Z}$ is surjective, which implies that $\rho: \pi \rightarrow S^{3}$ cannot factor through a proper quotient of $\pi$. The representation $\rho$ is thus faithful.

We will now make use of a locally finite approximation of $B S^{3} \simeq$ $B S L_{2}(\mathbb{C})$; by the result of [8] or [9] there exist a map

$$
\Phi: B S L_{2}\left(\bar{F}_{3}\right) \rightarrow B S^{3}
$$

such that $\Phi^{*}: H^{*}\left(B S^{3} ; Z / 2 Z\right) \cong H^{*}\left(B S L_{2}\left(\overline{\mathbb{F}}_{3}\right) ; \mathbb{Z} / 2 Z\right)$. As a consequence, $\Phi$ induces bijections

$$
\begin{aligned}
{\left[B S^{3},\left(B S^{3}\right)_{2}\right] } & \rightarrow\left[B S L_{2}\left(\overrightarrow{\mathbb{F}}_{3}\right),\left(B S^{3}\right)_{2}\right] \\
& \cong \underset{n}{\lim }\left[B S L_{2}\left(\mathbb{F}_{3^{n}}\right),\left(B S^{3}\right)_{2}\right]
\end{aligned}
$$


The second bijection stems from the observation that for $n>0$ the sets $\left[B S L_{2}\left(\mathbb{F}_{3^{n}}\right),\left(B S^{3}\right)_{2}^{\hat{2}}\right]$ have a natural compact topology (because $B S L_{2}\left(\mathbb{F}_{3^{n}}\right)$ is of the homotopy type of a space with finite skeleta and because the homotopy groups of $\left(B S^{3}\right)_{2}$ are compact abelian groups). It is well known that the groups $S L_{3}\left(\mathbb{F}_{3^{*}}\right)$ have periodic $Z / 2 Z$-cohomology, of period four [7]. The 2-subgroups of $S L_{2}\left(\mathbb{F}_{3^{n}}\right)$ are therefore cyclic or generalized quaternion groups. If $\pi$ is a 2-subgroup of $S L_{2}\left(\mathbb{F}_{3^{n}}\right)$, then the restriction map

$$
H^{4}\left(B S L_{2}\left(\mathbb{F}_{3^{n}}\right) ; Z\right) \rightarrow H^{4}(B \pi ; Z)=\mathbb{Z} /|\pi| Z
$$

is necessarily surjective, since a periodicity generator restricts to a periodicity generator. Consider now the map $\theta$ induced by $\Phi: B S L_{2}\left(\overline{\mathbb{F}}_{3}\right), \rightarrow B S^{3}$ followed by restriction to $B S L_{2}\left(\mathbb{F}_{3^{n}}\right), n \geqslant 1$,

$$
\theta:\left[B S^{3},\left(B S^{3}\right)_{2}^{\hat{n}}\right] \rightarrow \prod\left[B S L_{2}\left(\mathbb{F}_{3^{n}}\right),\left(B S^{3}\right)_{2}^{\hat{2}}\right]
$$

which, by (1.6), is injective. If the degree of $f: B S^{3} \rightarrow\left(B S^{3}\right)_{2}$ is a unit in $\mathbb{Z}_{2}$ and if $\pi \subset S L_{2}\left(\mathbb{F}_{3^{n}}\right)$ is a 2-subgroup then, by a slight variation of (1.5), we conclude that $\theta f \mid B \pi \simeq(B \rho)_{2}$, where $\rho: \pi \rightarrow S^{3}$ is a faithful representation. In order to prove part (ii) of (1.4) it suffices therefore to show that if $\pi(2, n) \subset S L_{2}\left(\mathbb{F}_{3^{n}}\right)$ denotes a 2-Sylow subgroup, then the restriction map

$$
\left[B S L_{2}\left(\mathbb{F}_{3^{n}}\right),\left(B S^{3}\right)_{2}\right] \rightarrow\left[B \pi(2, n),\left(B S^{3}\right)_{2}\right]
$$

is injective on the counter image of the set of all maps $B \pi(2, n) \rightarrow$ $\left(B S^{3}\right)_{2}$ which are of the form $(B \rho)_{2}$ for some faithful representation $\rho: \pi(2, n) \rightarrow S^{3}$. Theorem 1.4(ii) and thus the Classification Theorem is therefore a consequence of the following result.

THEOREM 1.7. Let $\pi(2, n) \subset S L_{2}\left(\mathbb{F}_{3^{n}}\right)$ denote a 2-Sylow subgroup, $n>0$. Consider the restriction map

$$
R:\left[B S L_{2}\left(\mathbb{F}_{3^{n}}\right),\left(B S^{3}\right)_{2}^{2}\right] \rightarrow\left[B \pi(2, n),\left(B S^{3}\right)_{2}^{\hat{n}}\right] .
$$

If $x \in\left[B S L_{2}\left(\mathbb{F}_{3^{n}}\right),\left(B S^{3}\right)_{2}\right]$ is an element such that $R(x)=(B \rho)_{2}$ for some faithful representation $\rho: \pi(2, n) \rightarrow S^{3}$ then $R^{-1} R(x)=\{x\}$.

The proof of this Theorem will be given in Section 3 .

\section{Functors on orbit categories}

Let $\pi$ be a finite group and let $O(\pi)$ denote the orbit category of $\pi$; its objects are $\pi$-sets of the form $\pi / \pi_{\alpha}, \pi_{\alpha} \subset \pi$ a subgroup, and morphisms $\pi / \pi_{\alpha} \rightarrow \pi / \pi_{\beta}$ are $\pi$-maps of the underlying $\pi$-set. If $X$ is a $\pi$-space and if $C_{*}\left(X^{\pi_{\sigma}}\right)$ denotes the singular chain complex of the fixed point set 
$X^{\pi_{*}} \subset X$, then

$$
\mathbf{C}_{i}(X)\left(\pi / \pi_{\alpha}\right)=C_{i}\left(X^{\pi_{\alpha}}\right)
$$

defines a contravariant functor $\mathrm{C}_{l}(X)$ on $O(\pi)$, with obvious values on morphisms. If we write $C(\pi)$, for the abelian category of contravariant functors $O(\pi) \rightarrow A b, A b$ the category of abelian groups, $\mathrm{C}_{*}(X)$ is a chain complex in $C(\pi)$, with respect to the obvious boundary operator. For any $\Phi \in C(\pi)$ the Bredon cohomology groups of $X$ with values in $\Phi$ are defined by

$$
H_{\pi}^{i}(X ; \Phi)=H^{i}\left(\operatorname{Hom}\left(\mathrm{C}_{*}(X), \Phi\right)\right)
$$

see [3] and [11]. Let $p$ be a prime and let $O_{p}(\pi)$ denote the full subcategory of $O(\pi)$ consisting of the objects of the form $\pi / \pi_{\alpha}$ where $\pi_{\alpha} \subset \pi$ is a $p$-subgroup. We write $C_{p}(\pi)$ for the corresponding category of contravariant functors $O_{p}(\pi) \rightarrow A b$. The inverse limit functor

$$
\lim : C_{p}(\pi) \rightarrow A b \text {, }
$$

which associates with $\Psi \in C_{p}(\pi)$ the inverse limit of the diagram of abelian groups $\left\{\Psi\left(\pi / \pi_{\alpha}\right)\right\}$ indexed by $O_{p}(\pi)^{\text {op }}$, is left exact and has right derived functors for $i \geqslant 1$

$$
\lim ^{i}=\operatorname{Ext}_{C_{(}(\pi)}^{i}(\mathbf{Z},): C_{p}(\pi) \rightarrow A b,
$$

where $\mathbf{Z}$ denotes the constant functor with value $\mathbf{Z}$. We call $\Psi \in C_{p}(\pi)$ acyclic, if $\lim ^{i} \Psi=0$ for ever $i>0$.

LeMma 2.1. Let $\Psi^{\prime} \in C_{p}(\pi), j \geqslant 0$, be defined by

$$
\Psi^{\prime}\left(\pi / \pi_{\alpha}\right)=H^{j}\left(E \pi \times_{\pi} \pi / \pi_{\alpha} ; \mathbb{Z} / p \mathbb{Z}\right)
$$

with obvious values on morphisms. Then $\Psi^{j}$ is acyclic.

Proof. It suffices to show that $\Psi=\oplus \Psi^{j}$ is acyclic. We write $\tilde{\Psi}$ for the obvious extension of $\Psi$ to the whole category $O(\pi)$. Thus

$$
\tilde{\Psi}\left(\pi / \pi_{\alpha}\right) \cong H^{*}\left(B \pi_{\alpha} ; \mathbb{Z} / p \mathbb{Z}\right)
$$

for $\pi / \pi_{\alpha} \in O(\pi)$. The ring structure of $H^{*}\left(B \pi_{\alpha} ; \mathbf{Z} / p \mathbf{Z}\right)$ gives rise to a pairing $\bar{\Psi} \times \bar{\Psi} \rightarrow \bar{\Psi}$ which, together with the usual transfer maps, make $\tilde{\Psi}$ into a Green functor, as defined in [4, Chapter 6]. Let $E \pi / p$ denote the universal $\pi$-space with respect to the family of $p$ subgroups of $\pi$ (cf. [4, Chapter 7]). The $\pi$-space $E \pi / p$ is characterized, up to a $\pi$-homotopy equivalence, by the fact that it is $\pi_{\alpha}$-contractible for every $p$-subgroup $\pi_{\alpha} \subset \pi$, and that the fixed point sets $(E \pi / p)^{H}$ are empty for every non-p-subgroup $H \subset \pi$. The projection $E \pi / p \rightarrow\left\{^{*}\right\}$ gives rise to a projective resolution $\mathbf{C}_{*}(E \pi / p) \rightarrow \mathbf{Z}$ in $C_{p}(\pi)$; the projectivity of $\mathbf{C}_{l}(E \pi / p)$ in $C_{p}(\pi)$ follows easily from the discussion of 
projectives in $C(\pi)$, (cf. [3]). We use the same notation for the functor $C_{i}(E \pi / p)$, considered as a functor on $C(\pi)$ or on $C_{p}(\pi)$. Therefore,

$$
\begin{aligned}
\lim ^{i} \Psi & =\operatorname{Ext}_{C_{\mathcal{P}(\pi)}}(\mathbf{Z}, \Psi) \\
& \cong H^{i}\left(\operatorname{Hom}_{C_{(}(\pi)}\left(\mathbf{C}_{*}(E \pi / p), \Psi\right)\right) \\
& \cong H_{\pi}^{i}(E \pi / p ; \tilde{\Psi})
\end{aligned}
$$

The last isomorphism follows from the observation that morphisms $\mathbf{C}_{i}(E \pi / p) \rightarrow \Psi$ in $C_{p}(\pi)$ are in one-to-one correspondence with morphisms $C_{i}(E \pi / p) \rightarrow \tilde{\Psi}$ in $C(\pi)$, because for ever non-p-subgroup $H \subset \pi$, $\mathbf{C}_{i}(E \pi / p)(\pi / H)=C_{l}\left((E \pi / p)^{H}\right)=0$ for $i \geqslant 0$. Let $\pi(p) \subset \pi$ denote a $p$-Sylow subgroup. Since the transfer map

$$
\operatorname{tr}: H^{*}(B \pi(p) ; \mathbf{Z} / p \mathbf{Z}) \rightarrow H^{*}(B \pi ; \mathbb{Z} / p \mathbb{Z})
$$

is surjective, the Green functor $\bar{\Psi}$ satisfies induction with respect to $p$-subgroups. The transfer map in Bredon cohomology, as defined in [11],

$$
\text { tr: } H_{\pi(p)}^{i}(E \pi / p ; \tilde{\Psi} \mid O(\pi(p))) \rightarrow H_{\pi}^{i}(E \pi / p ; \tilde{\Psi})
$$

is therefore surjective for every $i \geqslant 0$. But $H_{\pi(p)}^{i}(E \pi / p ; \tilde{\Psi} \mid O(\pi(p)))=0$ for $i>0$ because $E \pi / p$ is $\pi(p)$-contractible. Consequently, the identifications (2.2) show that $\lim ^{i} \Psi=0$ for every $i>0$.

\section{The proof of Theorem 1.7}

Let $\pi$ be a finite group. Consider the functor $F$ from $O_{p}(\pi)$ to spaces, associating with $\pi / \pi_{\alpha} \in O_{p}(\pi)$ the space $E \pi \times_{\pi} \pi / \pi_{\alpha}$, with obvious values on morphisms. We will write

$$
\operatorname{hocolim}\left(E \pi \times_{\pi} \pi / \pi_{\alpha}\right)
$$

for the homotopy direct limit of $F$ in the sense of [1]. This space, which is closely related to the space $\pi \backslash E(\pi / \pi(p))$ of [12] enjoys the following property.

\section{LEMMA 3.1. The natural map}

$$
L: \operatorname{hocolim}\left(E \pi \times_{\pi} \pi / \pi_{\alpha}\right) \rightarrow B \pi
$$

induced by the projections $E \pi \times_{\pi}\left(\pi / \pi_{\alpha}\right) \rightarrow E \pi \times_{\pi}(\pi / \pi)=B \pi$ is a $H_{*}(; \mathbf{Z} / p \mathbf{Z})$-isomorphism.

Proof. The hocolim-spectral sequence of [1, XII 4.5] takes the form $E_{2}^{i, j}=\lim ^{i} H^{j-i}\left(\left(E \pi \times_{\pi} \pi / \pi_{\alpha}\right) ; \mathbf{Z} / p \mathbb{Z}\right) \Rightarrow H^{j}\left(\operatorname{hocolim}\left(E \pi \times_{\pi} \pi / \pi_{\alpha}\right) ; \mathbf{Z} / p \mathbf{Z}\right)$ By (2.1), $E_{2}^{i, j}=0$ for $i \neq 0$, and the spectral sequence collapses to yield 
the isomorphism

$$
H^{i}\left(\operatorname{hocolim}\left(E \pi \times_{\pi} \pi / \pi_{\alpha}\right) ; \mathbf{Z} / p \mathbf{Z}\right) \stackrel{\rightarrow}{\rightarrow} \lim H^{i}\left(E \pi \times_{\pi} \pi / \pi_{\alpha} ; \mathbf{Z} / p \mathbf{Z}\right)
$$

On the other hand it is a classical fact that restriction to $p$-subgroups induces an isomorphism

$$
H^{i}(B \pi ; \mathbb{Z} / p \mathbb{Z}) \leftrightarrows \lim H^{i}\left(B \pi_{\alpha} ; \mathbf{Z} / p \mathbb{Z}\right) \cong \lim H^{i}\left(E \pi \times_{\pi} \pi / \pi_{\alpha} ; \mathbf{Z} / p \mathbb{Z}\right)
$$

and it follows then that $L$ is a $H_{*}(; \mathbb{Z} / p Z)$-isomorphism.

We consider now the case of $\pi=S L_{2}\left(\mathbb{F}_{3^{n}}\right)$ and $p=2$. Let $x \in$ $\left[B \pi,\left(B S^{3}\right)_{2}\right]$ be as in Theorem 1.7. As observed, the restriction $x \mid B \pi_{\alpha}$ for $\pi_{\alpha} \subset \pi$ a subgroup is of the form $\left(B \rho_{\alpha}\right)_{2}$, where $\rho_{\alpha}: \pi_{\alpha} \rightarrow S^{3}$ is a faithful representation, uniquely determined up to conjugation by $x$. We write

$$
X_{\alpha}=\operatorname{map}\left(E \pi \times_{\pi} \pi / \pi_{\alpha},\left(B S^{3}\right)_{2}\right)_{\rho_{\alpha}}
$$

for the component of the space of all (free) maps $E \pi \times_{\pi} \pi / \pi_{\alpha} \rightarrow\left(B S^{3}\right)_{2}$ containing the map $\left(B \rho_{\alpha}\right)_{2}$. Because $\left(B S^{3}\right)_{2}$ is simply connected and $\mathbb{Z} / 2$-complete, the map $L$ of (3.1) induces a homotopy equivalence

$$
L^{*}: \operatorname{map}\left(B \pi,\left(B S^{3}\right)_{2}\right) \underset{m}{\longrightarrow} \operatorname{map}\left(\operatorname{hocolim}\left(E \pi \times_{\pi} \pi / \pi_{\alpha}\right),\left(B S^{3}\right)_{2}\right)
$$

Consider now the diagram

$$
\begin{gathered}
X=\operatorname{holim} X_{\alpha} \subset \operatorname{holim}\left(\operatorname{map}\left(E \pi \times_{\pi} \pi / \pi_{\alpha},\left(B S^{3}\right)_{2}\right)\right) \\
\operatorname{map}\left(\operatorname{hocolim}\left(E \pi \times_{\pi} \pi / \pi_{\alpha}\right),\left(B S^{3}\right)_{2}\right) \\
\operatorname{map}\left(B \pi,\left(B S^{3}\right)_{2}\right) .
\end{gathered}
$$

It shows that the space $X$ is homotopy equivalent to the union of all those components of map $\left(B \pi,\left(B S^{3}\right)_{2}\right)$ which contain the maps $f: B \pi \rightarrow$ $\left(B S^{3}\right)_{2}^{2}$ whose restrictions satisfy $f \mid B \pi_{\alpha} \simeq\left(B \rho_{\alpha}\right)_{2}$ for every 2-subgroup $\pi_{\alpha} \subset \pi$. Proving (1.7) amounts therefore to showing that the space $X$ is connected. This will be achieved by computing $H_{0}(X)$, using the strong convergence theorem for the homology spectral sequence for homotopy inverse limits, as proved by A. K. Bousfield in [2]. The spaces $X_{\alpha}$ which make up $X$ have the following structure.

Lemma 3.2. The homotopy types $X_{\alpha}$ depend in the following way on $\pi / \pi_{\alpha} \in O_{2}(\pi)$ :

(i) if $\left|\pi_{\alpha}\right| \leqslant 2$, then $X_{\alpha} \simeq\left(\mathbb{H} P P^{\infty}\right)_{2}$

(ii) if $\left|\pi_{\alpha}\right|>2, \pi_{\alpha}$ abelian, then $X_{\alpha} \approx\left(\mathbb{C} P^{\infty}\right)_{2}$

(iii) if $\pi_{\alpha}$ is non-abelian, then $X_{\alpha} \simeq \mathbb{R} P^{\infty}$. 
Proof. By definition $X_{\alpha}=\operatorname{map}\left(E \pi \times_{\pi} \pi / \pi_{\alpha},\left(B S^{3}\right)_{2}\right)_{\rho_{\alpha}} \quad$ where $\rho_{\alpha}: \pi_{\alpha} \rightarrow S^{3}$ is faithful. In case $\left|\pi_{\alpha}\right| \leqslant 2, \rho\left(\pi_{\alpha}\right) \subset S^{3}$ is central and therefore the centralizer $C\left(\rho_{\alpha}\right)$ is $S^{3}$. If $\pi_{\alpha}$ is abelian of order $\geqslant 4$, $C\left(\rho_{\alpha}\right) \subset S^{3}$ is a maximal torus $S^{1}$, and if $\pi_{\alpha}$ is non-abelian, it is easy to see that $C\left(\rho_{\alpha}\right)$ is the center of $S^{3}, C\left(\rho_{\alpha}\right) \simeq \mathbb{Z} / 2 \mathbb{Z}$. In either case $B C\left(\rho_{\alpha}\right)$ is a simple space of finite type. Proposition (ii) implies therefore that

$$
\Phi\left(\rho_{\alpha}\right)_{2}:\left(B C\left(\rho_{\alpha}\right)\right)_{2} \stackrel{a}{\longrightarrow} \operatorname{map}\left(B \pi_{\alpha},\left(B S^{3}\right)_{2}\right)_{\rho_{\alpha}}
$$

Hence $X_{\alpha} \simeq\left(B C\left(\rho_{\alpha}\right)\right)_{2}$ has the form stated.

In particular, all the homotopy groups of the spaces $X_{\alpha}$ are abelian and for every $i>0$ we have thus well-defined contravariant functors

$$
\Phi(i): O_{2}(\pi) \rightarrow A b
$$

given by $\Phi(i)\left(\pi / \pi_{\alpha}\right)=\pi_{i}\left(X_{\alpha}\right)$, with morphisms $\pi / \pi_{\alpha} \rightarrow \pi / \pi_{\beta}$ inducing maps $\pi_{i}\left(X_{\beta}\right) \rightarrow \pi_{i}\left(X_{\alpha}\right)$ which are easy to calculate.

THEOREM 3.3. The functors $\Phi(i)$ are acyclic for all $i>0$.

Proof. There are the following three cases to consider.

(I) $\Phi(1)$. $\Phi(1)\left(\pi / \pi_{\alpha}\right)=\pi_{1}\left(X_{\alpha}\right)$ which, by (3.2), is $Z / 2 Z$, if $\pi_{\alpha}$ is non-abelian, and 0 in all other cases. The morphisms $\Phi(1)\left(\pi / \pi_{\alpha} \rightarrow \pi / \pi_{\beta}\right)$ are isomorphisms if $\pi_{\alpha}$ and $\pi_{\beta}$ are non-abelian.

(II) $\Phi(2) . \Phi(2)\left(\pi / \pi_{\alpha}\right)=\pi_{2}\left(X_{\alpha}\right)$ which is $\hat{Z}_{2}$ if $\pi_{\alpha}$ is cyclic of order at least four, and 0 in all other cases. Note that there are obvious natural transformations $\Phi(2) \rightarrow \Phi(2) / 2^{j} \Phi(2), j \geqslant 1$, and

$$
\Phi(2)=\lim _{j} \Phi(2) / 2^{\prime} \Phi(2)
$$

in $C_{2}(\pi)$. Moreover, $\lim _{\leftarrow}^{1} \Phi(2) / 2^{2} \Phi(2)=0$ because the groups $(\Phi(2) /$ $\left.2^{j} \Phi(2)\right)\left(\pi / \pi_{\alpha}\right)$ are all finite. Therefore, there is a short exact sequence

$$
\Phi(2) \rightarrow \prod_{j} \Phi(2) / 2^{j} \Phi(2) \rightarrow \prod_{j} \Phi(2) / 2^{j} \Phi(2)
$$

in $C_{2}(\pi)$. Observe that for every $j$

$$
\lim _{\left(\pi / \pi_{\alpha} \in O_{2}(\pi)^{\text {का })}\right.}\left(\Phi(2) / 2^{j} \Phi(2)\right)\left(\pi / \pi_{\alpha}\right)=0
$$

because $\Phi(2)\left(\pi / \pi_{\beta}\right)=0$ if $\pi_{\beta}$ is non-abelian, and for every $\pi / \pi_{\alpha} \in O_{2}(\pi)$ there is a morphism $\pi / \pi_{\alpha} \rightarrow \pi / \pi_{\beta}$ with $\pi_{\beta}$ non-abelian. Clearly, if $\Phi(2) / 2 \Phi(2)$ is acyclic then so are all the functors $\Phi(2) / 2 \boldsymbol{J} \Phi(2)$. Using (3.4) and (3.5) we see that if $\Phi(2) / 2 \Phi(2)$ is acyclic then so is $\Phi(2)$. We will write $\Phi(2)$ for $\Phi(2) / 2 \Phi(2)$. Thus $\overline{\Phi(2)}\left(\pi / \pi_{\alpha}\right)=Z / 2 Z$ if $\pi_{\alpha} \cong Z / 2^{n} Z$, $n \geqslant 2$, and $\overline{\Phi(2)}\left(\pi / \pi_{\alpha}\right)=0$ in all other cases. Note also that a morphism 
$f: \pi / \pi_{\alpha} \rightarrow \pi / \pi_{\beta}$ induces an isomorphism $\pi_{2}\left(X_{\beta}\right) \rightarrow \pi_{2}\left(X_{\alpha}\right)$ if these groups are non-trivial, and therefore $\overline{\Phi(2)}(f)$ is an isomorphism if $\overline{\Phi(2)}\left(\pi / \pi_{\alpha}\right)$ and $\overline{\Phi(2)}\left(\pi / \pi_{\beta}\right)$ are cyclic of order two.

(III) $\Phi(i)$ for $i>2$. $\Phi(i)\left(\pi / \pi_{\alpha}\right)=\pi_{i}\left(X_{\alpha}\right)=0$ if $\left|\pi_{\alpha}\right|>2$ and $\Phi(i)(\pi /$ $\left.\pi_{\alpha}\right) \cong \pi_{i-1}\left(S^{3}\right) \otimes \mathbf{Z}_{2} \cong A$ if $\pi_{\alpha} \cong \mathbf{Z} / 2 Z$ or $\{1\}$. The induced morphisms $A \rightarrow A$ are easily all seen to be equivalent to the identity. Since $A$ is a finitely generated $\mathbf{Z}_{2}$-module one can argue as in case (II) to show that it suffices to consider the case $A=Z / 2 Z$ to take care of (III) in general. Note that $A=\mathbf{Z} / 2 Z$ corresponds to the case of $\Phi(5)$.

We will prove the acyclicity of $\Phi(1), \overline{\Phi(2)}$ and $\Phi(5)$ by relating these functors to the acyclic functors $\Psi^{j} \in C_{2}(\pi)$ of (2.1), which satisfy $\Psi^{\prime}\left(\pi / \pi_{\alpha}\right) \cong H^{i}\left(B \pi_{\alpha} ; Z / 2 Z\right), \pi=S L_{2}\left(F_{3^{n}}\right)$ as above. Any morphism $\pi /$

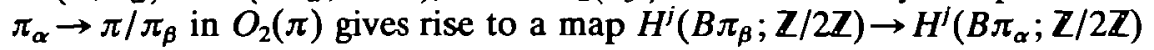
which is of the form in $(g)^{*}$, where in $(g): \pi_{\alpha} \rightarrow \pi_{\beta}$ is given by in $(g)(x)=$ $g x g^{-1}$ for some $g \in \pi$. In the discussion which follows it will suffice to understand the cohomological restriction maps associated to inclusions $\pi_{\alpha} \subset \pi_{\beta}$ in order to understand the more general in $(g)^{*}$.

Splitting of $\Psi^{1}$. If $f: \pi / \pi_{\alpha} \rightarrow \pi / \pi_{\beta}$ is a morphism in $O_{2}(\pi)$ with $\pi_{\alpha} \cong \mathbb{Z} / 2 \mathbb{Z}$ and $\left|\pi_{\beta}\right|>2$, then $\Psi^{1}(f)=0$, because the cohomological restriction map for $\mathbf{Z} / 2 \mathbb{Z} \subset \pi_{\beta}$, res: $H^{i}\left(B \pi_{\beta} ; \mathbf{Z} / 2 Z\right) \rightarrow H^{i}(B \mathbf{Z} / 2 \mathbf{Z} ; \mathbf{Z} / 2 \mathbb{Z})$, is zero (it suffices to consider the case of $Z / 2 Z \subset Z / 4 Z$ ). Hence we can write $\Psi^{1}$ as sum of two acyclic functors

$$
\Psi^{1}=\Psi^{11} \oplus \Psi^{12}
$$

where $\Psi^{11}\left(\pi / \pi_{\alpha}\right)=0$ unless $\pi_{\alpha} \cong Z / 2 Z$, in which latter case it takes the value $\mathbf{Z} / 2 \mathbf{Z}$.

Splitting of $\Psi^{2}$. Let $Z / 2^{n-1} Z \subset Q_{2^{n}}$ be a maximal cyclic subgroup, $n \geqslant 3$ and $Q_{Z^{n}}$ the generalized quaternion group of order $2^{n}$. The restriction map $H^{2}\left(B Q_{Z^{n}} ; \mathbf{Z} / 2 \mathbf{Z}\right) \rightarrow H^{2}\left(B\left(Z / 2^{n-1} \mathbf{Z}\right) ; \mathbf{Z} / 2 Z\right)$ is zero, because it is the reduction $\bmod 2$ of a map $Z / 2 Z \oplus Z / 2 Z \rightarrow Z / 2^{n-1} Z, n \geqslant 3$. We conclude that

$$
\Psi^{2}=\Psi^{21} \oplus \Psi^{22}
$$

where $\Psi^{21}\left(\pi / \pi_{\alpha}\right) \cong H^{2}\left(B \pi_{\alpha} ; \mathbf{Z} / 2 \mathbf{Z}\right) \cong \mathbf{Z} / 2 \mathbf{Z}$ for every non-zero cyclic group $\pi_{\alpha}$, and $\Psi^{21}\left(\pi / \pi_{\alpha}\right)=0$ if $\pi_{\alpha}$ is not cyclic. Since the restriction map $H^{2}\left(B\left(\mathbb{Z} / 2^{n} \mathbf{Z}\right), \mathbf{Z} / 2 \mathbb{Z}\right) \rightarrow H^{2}\left(B\left(\mathbf{Z} / 2^{n-1} \mathbf{Z}\right) ; \mathbf{Z} / 2 \mathbf{Z}\right)$ is an isomorphism for $n \geqslant$ 2 , we infer that every $f: \pi / \pi_{\alpha} \rightarrow \pi / \pi_{\beta}$ with $\pi_{\alpha}, \pi_{\beta}$ cyclic and non-trivial, induces an isomorphism $\Psi^{21}(f)$.

Splitting of $\Psi^{4}$. Every $f: \pi / \pi_{\alpha} \rightarrow \pi / \pi_{\beta}$ with $\pi_{\alpha} \neq\{1\}$ induces an isomorphism $\Psi^{4}(f): \mathbb{Z} / 2 Z \rightarrow Z Z / 2 Z$, since a periodicity generator in $H^{4}$ restrict to a periodicity generator. From the description of $\Psi^{21}$ it is thus clear that there is a short exact sequence in $C_{2}(\pi)$ of the form

$$
\Psi^{21} \rightarrow \Psi^{4} \rightarrow \Psi^{4} / \Psi^{21}
$$


The associated long exact sequence

$$
0 \rightarrow \lim \Psi^{21} \rightarrow \lim \Psi^{4} \rightarrow \lim \Psi^{4} / \Psi^{21} \rightarrow \lim ^{1} \Psi^{21} \rightarrow \cdots
$$

shows that $\Psi^{4} / \Psi^{21}$ is acyclic, since $\Psi^{21}$ and $\Psi^{4}$ are acyclic.

Acyclicity of $\Phi(1)$. From the description of $\Phi(1)$ it follows that it is isomorphic to $\Psi^{4} / \Psi^{21}$, which we just proved to be acyclic.

Acyclicity of $\overline{\Phi(2)}$. In this case we consider the obvious short exact sequence of functors

$$
\Psi^{11} \rightarrow \Psi^{21} \rightarrow \overline{\Phi(2)}
$$

with $\Psi^{11}$ and $\Psi^{21}$ acyclic. As argued above, the associated long exact sequence of derived functors of lim then implies that $\overline{\Phi(2)}$ is acyclic.

Acyclicity of $\Phi(5)$. The constant functor $\Psi^{0}$ satisfies $\Psi^{0}\left(\pi / \tilde{\pi}_{\alpha}\right)=\mathbf{Z} / 2 Z$ for every $\pi / \pi_{\alpha}$ in $O_{2}(\pi)$ and it maps onto $\Psi^{4}$ with kernel a functor $\Psi^{04}$ such that $\Psi^{04}\left(\pi / \pi_{\alpha}\right) \cong \mathbb{Z} / 2 Z$ if $\pi_{\alpha}=\{1\}$, and $\Psi^{04}\left(\pi / \pi_{\alpha}\right)=0$ if $\left|\pi_{\alpha}\right|>1$. Thus we have a short exact sequence in $O_{2}(\pi)$ of the form

$$
\Psi^{04} \rightarrow \Psi^{0} \rightarrow \Psi^{4}
$$

Since $\Psi^{0}\left(\pi / \pi_{\alpha}\right) \rightarrow \Psi^{4}\left(\pi / \pi_{\alpha}\right)$ is an isomorphism if $\left|\pi_{\alpha}\right|>1$, it follows that the induced homomorphism $\lim \Psi^{0} \rightarrow \lim \Psi^{4}$ is an isomorphism $Z / 2 Z \rightarrow$ $Z / 2 Z$. Hence, from the acyclicity of $\Psi^{0}$ and $\Psi^{4}$, we infer that $\Psi^{04}$ is acyclic. The definition of $\Phi(5)$ shows that there is a short exact sequence

$$
\Psi^{04} \rightarrow \Phi(5) \rightarrow \Psi^{11}
$$

and therefore $\Phi(5)$ is acyclic. This completes the proof of Theorem 3.3.

We return now to the proof of Theorem 1.7. Recall that we have to show that $X=\operatorname{holim}\left(X_{\alpha}\right)$ is connected. According to (3.2) each $X_{\alpha}$ is connected and simple, and by (3.3)

$$
\lim ^{m} \pi_{m+n}\left(X_{\alpha}\right)=\lim ^{m} \Phi(m+n)=0
$$

for every $m>0$. It follows therefore from $[2,4.3]$ that there is a strongly convergent spectral sequence

$$
E_{s, t}^{2}=\pi^{s} H_{t}\left(\Pi^{*}\left\{X_{\alpha}\right\} ; Z / 3 Z\right) \Rightarrow H_{t-s}(X ; Z / 3 Z)
$$

where $\Pi^{*}\left\{X_{\alpha}\right\}$ denotes the cosimplicial space associated with the diagram $\left\{X_{\alpha}\right\}$. Each $X_{\alpha}$ is $Z / 3 Z$-acyclic by (3.2). Therefore, $E_{s, t}^{2}=0$ if $(s, t) \neq(0,0)$ and $E_{0,0}^{2} \cong Z / 3 Z$, which implies that the spectral sequence collapses to give $H_{0}(X ; Z / 3 Z) \cong Z / 3 Z$. It follows that $X$ is connected, completing the proof of the Classification Theorem.

Remark. We leave it to the reader to check that in a similar (but much less involved) fashion one would obtain an other proof of 1.4(i), if one works with an odd prime instead of the prime 2 . 


\section{Appendix}

The following is a proof in the spirit of this paper of the fact [6] that the degree of a self-map of $B S^{3}$ is zero or an odd square.

Let $f: B S^{3} \rightarrow B S^{3}$ be given. We first show that $\operatorname{deg}(f)$ is a square. It obviously suffices to show that $\operatorname{deg}(f)$ is a square $\bmod p^{\alpha}$ for every prime power $p^{\alpha}$. For this consider $\mathbf{Z} / p^{\alpha} \mathbf{Z} \subset S^{3}$, a cyclic subgroup of order $p^{\alpha}$. Since $f \mid B \mathbb{Z} / p^{\alpha}=B \rho(\alpha)$ for some representation $\rho(\alpha): \mathbb{Z} / p^{\alpha} \rightarrow S^{3}$, we see, by considering the second Chern class of $\rho(\alpha)$, that

$$
(B \rho(\alpha))^{*} x=k^{2} y=\operatorname{deg}(f) \cdot y \in H^{4}\left(\mathbb{Z} / p^{\alpha} \mathbf{Z} ; Z\right)
$$

where $x \in H^{4}\left(B S^{3} ; \mathbf{Z}\right)$ denotes a generator and $y \in H^{4}\left(Z / p^{\alpha} \mathbf{Z} ; \mathbb{Z}\right)$ the restriction of $x$. Since $y$ generates $H^{4}\left(Z / p^{\alpha} ; Z\right) \cong Z / p^{\alpha} Z$ it follows that $\operatorname{deg}(f)=k^{2} \bmod p^{\alpha}$. It remains to show that $\operatorname{deg}(f)$ is odd, if it is non-zero. Consider

$$
\mathrm{Z} / 4 \mathbf{Z} \subset Q_{2^{k}} \subset S^{3}
$$

where $Q_{2^{k}}$ is a generalized quaterion group of order $2^{k} \geqslant 8$. Let us write $\operatorname{deg}(f)=2^{n} m$ with $m$ odd and $n \geqslant 1$. Put $f \mid B Q_{2^{k}}=B \sigma(k), \sigma(k): Q_{2^{k}} \rightarrow$ $S^{3}$ a representation. Then, since $\operatorname{deg}(f)$ is a square, $B(\sigma(k) \mid(Z)$ $4 Z))^{*} x=0$ for $x \in H^{4}\left(B S^{3} ; Z\right)$ a generator, which implies that $\sigma(k)$ is not faithful. Hence $\sigma(k)$ factors through the centre of $S^{3}$, since the only proper non-trivial quotient of $Q_{2^{k}}$ with periodic cohomology is $Z / 2 Z$. But this implies that

$$
(B \sigma(k) \mid M)^{*} x=0 \in H^{4}(M ; \mathbb{Z})
$$

where $M \subset Q_{2^{k}}$ denotes a suitable maximal subgroup. Hence, $\operatorname{deg}(f)=0 \bmod 2^{k-1}$ by the argument above. Since this holds for any $k \geqslant 3$ we obtain a contradiction, showing that $\operatorname{deg}(f)=m$ is odd.

\section{REFERENCES}

1. A. K. Bousfield and D. M. Kan, Homotopy limits, completions and localizations. Lecture Notes in Math. 304, Berlin-Heidelberg-New York: Springer 1972.

2. A. K. Bousfield, 'On the homology spectral sequence of a cosimplicial space', Preprint.

3. G. E. Bredon, Equivariant cohomology theories. Lecture Notes in Math. 34, Berlin-Heidelberg-New York: Springer 1967.

4. T. Tom Dieck, Transformation groups and representation theory. Lecture Notes in Math. 766, Berlin-Heidelberg-New York: Springer 1979.

5. W. G. Dwyer, 'Maps between classifying spaces', Preprint.

6. S. Feder and S. Gitler, 'Mappings of quaternionic projective spaces', Bol. de la Soc. Mat. Mex. 18 1973, (33-37).

7. Z. Fiedorowice and S. Priddy, Homology of classical groups over finite fields and their associated infinite loop spaces. Lecture Notes in Math. 674, Berlin-Heidelberg-New York: Springer 1978. 
8. E. M. Friedlander and G. Mislin, 'Cohomology of classifying spaces of complex Lie group and related discrete groups', Comment. Math. Helv. 59 (1984), 347-361.

9. E. M. Friedlander and G. Mislin, 'Locally finite approximation of Lie groups I', Inventiones Math. 83 (1986), 425-436.

10. E. M. Friedlander and G. Mislin, 'Locally finite approximation of Lie groups II', Math. Proc. Camb. Phil. Soc. 100 (1986), 505-517.

11. S. Ilman, Equivariant singular homology and cohomology I. American Math. Society, Memoirs No. 156, 1975.

12. H. Miller, 'The Sullivan conjecture on maps from classifying spaces', Annals of Math. 120 (1984), 39-87.

13. H. Miller, To appear.

14. D. Sullivan, 'Genetics in homotopy theory and the Adams conjecture', Ann. of Mathematics 100 (1974), 1-79.

15. Z. Wojtkowiak, 'When a map equivariant up to homotopy is homotopic to an equivariant one, Preprint.

16. A. Zabrodsky, 'Maps between classifying spaces', To appear.

Ohio State University

Department of Mathematics

Columbus, Ohio 43210

U.S.A.

Mathematik

ETH-Zentrum

8092-Zurich

Switzerland 